\title{
Editorial
}

\section{Materials Chemistry for Sustainability and Energy}

\author{
Fan Dong, ${ }^{1}$ Sirilak Sattayasamitsathit, ${ }^{2}$ Yu Xin Zhang, ${ }^{3}$ and Ying Zhou ${ }^{4}$ \\ ${ }^{1}$ Chongqing Key Laboratory of Catalysis and Functional Organic Molecules, College of Environmental and Biological Engineering, \\ Chongqing Technology and Business University, Chongqing 400067, China \\ ${ }^{2}$ Department of Nanoengineering, University of California, San Diego (UCSD), La Jolla, CA 92093, USA \\ ${ }^{3}$ Department of Materials Science and Engineering, Chongqing University, Chongqing 400044, China \\ ${ }^{4}$ School of Materials Science and Engineering, State Key Laboratory of Oil and Gas Reservoir Geology and Exploitation, \\ Southwest Petroleum University, Chengdu 610500, China
}

Correspondence should be addressed to Fan Dong; dfctbu@126.com

Received 22 September 2014; Accepted 22 September 2014; Published 22 December 2014

Copyright (C) 2014 Fan Dong et al. This is an open access article distributed under the Creative Commons Attribution License, which permits unrestricted use, distribution, and reproduction in any medium, provided the original work is properly cited.

Environmental pollution and energy storage are two global menaces and the magnitude of them is ever-increasing due to rapid pace of urbanization and industrialization. Thus, providing clean environment and renewable energy for people is a great challenge. The nano/microstructured materials with intriguing physical and chemical properties have given immense scope and opportunities to address the key issues of environmental sustainability and clean energy development. In the past two decades, great progress has been made on design, synthesis, and utilization of artificial materials for sustainability and energy. These nano/microstructured materials in various fashions are stimulating various practical applications in environmental and energy sector. The rapid development in materials chemistry has led to significant advances in understanding the controlled synthesis, chemical properties, and structure-activity relationship of materials.

This special issue contains 13 papers, which are mainly related to energy development and pollutant removal utilizing newly artificial materials. Among them, 5 papers are related to pollutants removal, 3 papers are about batteries development, and 2 papers are for solar cell. In addition, 1 paper is related to investigations of the montmorillonite and aluminium trihydrate addition effects on the ignitability and thermal stability of asphalt and 1 paper is exploring the effects of concentration, temperature, torque value, roughness, and washer shape on crevice corrosion for 254 SMO alloys. Furthermore, 1 paper is about newly polymeric anion-exchange membranes (AEMs) fabrication. Additionally, there is 1 review that systematically introduces the synthesis and application of one-dimensional $\mathrm{La}(\mathrm{OH})_{3}$ nanostructures. We would like to express our sincere thanks to all the authors for submitting their interesting and meaningful works to this special issue. A brief summary of all 13 papers is provided below.

The paper entitled "A quaternized polysulfone membrane for zinc-bromine redox flow battery" reported that a quaternized polysulfone (QNPSU) composite membrane was synthesized for zinc-bromine redox flow battery. The conductivity of the membrane was tested by electrochemical analyzer. It was found that the conductivity of the membrane was about $0.01 \mathrm{~S} / \mathrm{cm}$ in $25^{\circ} \mathrm{C}$ with $100 \mathrm{RH} \%$. Furthermore, the discharge voltage was $0.9672 \mathrm{~V}$ and the power density was $6 \mathrm{~mW} / \mathrm{cm}^{2}$ at a current of $0.1 \mathrm{~A}$, which demonstrated that the novel composite membrane was a promising material for the flow battery.

In "A study on the morphology of a dispersed particle gel used as a profile control agent for improved oil recovery," a new profile control agent termed dispersed particle gel (DPG) has been fabricated and reported, which was used to control the injection water and improve oil recovery. The AFM studies showed that DPG was composed of small pseudospherical particles and that their sizes can be controlled by adjusting the initial polymer mass concentration, the shearing rate, and the salinity. Furthermore, the effects of the initial polymer mass concentration, the shearing rate, the salinity, and the hightemperature aging on the particle size of DPG were studied 
by dynamic light scattering (DLS). This work paves the way for the formula design of DPG and its application in the oil and gas field.

The paper "Catalytic combustion of low concentration methane over catalysts prepared from $\mathrm{Co} / \mathrm{Mg}-\mathrm{Mn}$ layered double hydroxides" described that a series of $\mathrm{Co} / \mathrm{Mg}-\mathrm{Mn}$ mixed oxides were synthesized through thermal decomposition of layered double hydroxides (LDHs) precursors. The resulted catalysts were then subjected for catalytic combustion of methane and it was revealed that the $\mathrm{Co}_{4.5} \mathrm{Mg}_{1.5} \mathrm{Mn}_{2} \mathrm{LDO}$ catalyst possessed the best performance with the $\mathrm{T}_{90}=$ $485^{\circ} \mathrm{C}$. The redox ability of the catalysts can be significantly improved by the addition of cobalt. Besides, certain amount of magnesium was essential to guarantee the catalytic activity, which was helpful to enhance the oxygen mobility and improve the dispersion of $\mathrm{Co}$ and $\mathrm{Mn}$ oxides, preventing the surface area loss after calcination.

The paper entitled "Development of low energy gap and fully regioregular polythienylenevinylene derivative" introduced that low energy gap and fully regioregular conjugated polymers were widely used in solar energy conversion applications. This paper reported the synthesis and characterization of a new polymer, a low energy gap, fully regioregular, terminal functionalized, and processable conjugated polymer poly-(3-dodecyloxy-2,5-thienylene vinylene) or PDDTV. The UV-vis-NIR absorption spectrum demonstrated that the polymer exhibited an optical energy gap of $1.46 \mathrm{eV}$. The highest occupied molecular orbital (HOMO) level is $-4.79 \mathrm{eV}$ based on electrochemical measurement, while the lowest unoccupied molecular orbital (LUMO) level is $-3.33 \mathrm{eV}$ based on optical energy gap. The polymer is thermally stable up to about $300^{\circ} \mathrm{C}$, which demonstrated that this polymer is beneficial for effective solar cell applications.

The paper "Inhibition effect of glycerol on the corrosion of copper in $\mathrm{NaCl}$ solutions at different $\mathrm{pH}$ values" investigated the inhibition effect of glycerol against copper corrosion in aerated $\mathrm{NaCl}(0.5 \mathrm{M})$ solution at various $\mathrm{pH}$ values. The inhibition efficiency was assessed by conventional electrochemical techniques: open circuit potential, potentiodynamic polarization, and electrochemical impedance analysis. It was found that glycerol is capable of reducing the corrosion rate of copper in $\mathrm{NaCl}$ solutions. Moreover, glycerol was more efficient for use in alkaline chloride media where the best inhibition effect was obtained $(\eta \approx 83 \%)$. The inhibition efficiency in alkaline media was ascribed to an increase in viscosity and the presence of copper-glycerol complexes.

The paper entitled "Investigations of the montmorillonite and aluminium trihydrate addition effects on the ignitability and thermal stability of asphalt" investigated the effects of unmodified montmorillonite (MMT), organically modified montmorillonite (OMMT), and aluminium trihydrate (ATH) additions on the flame retardancy for asphalt combustion by means of limiting oxygen index (LOI), cone calorimeter, and TG-DSC tests. Experimental results revealed that adding a small amount of montmorillonite did not significantly increase the oxygen index of the asphalt. Besides, TGA measurements showed that the montmorillonite (MMT, OMMT) could suppress the release of flammable volatiles and form more asphaltenes, which hence postponed the burnout time of asphalt. In addition, the combining montmorillonite (MMT, OMMT) and ATH exerted a synergistic effect, which further reduced the heat release rate and increased the oxygen index of asphalt simultaneously.

In "Phthaloylchitosan-based gel polymer electrolytes for efficient dye-sensitized solar cells," phthaloylchitosan-based gel polymer electrolytes were fabricated with tetrapropylammonium iodide $\left(\mathrm{Pr}_{4} \mathrm{NI}\right)$, a material with decent conductivity. The electrolyte with the composition rate of phthaloylchitosan (15.7 wt.\%), ethylene carbonate (EC) (31.7 wt.\%), propylene carbonate (PC) (3.17 wt.\%), $\mathrm{Pr}_{4} \mathrm{NI}$ (19.0 wt.\%), and iodine (1.9 wt.\%) exhibited the highest room temperature ionic conductivity of $5.27 \times 10^{-3} \mathrm{~S} \mathrm{~cm}^{-1}$. The dye-sensitized solar cell (DSSC) fabricated with this electrolyte exhibited an efficiency of $3.5 \%$ with JSC of $7.38 \mathrm{~mA} \mathrm{~cm}^{-2}$, VOC of $0.72 \mathrm{~V}$, and fill factor of 0.66 . The overall conductivity was observed to decrease as various amounts of lithium iodide (LiI) were added to the optimized gel electrolyte.

The paper entitled "Preparation of mesoporous $\mathrm{SnO}_{2}$ by electrostatic self-assembly" described that mesoporous $\mathrm{SnO}_{2}$ nanoparticles with diameters of 5-6 nm crystalline walls and 3-4 $\mathrm{nm}$ pore diameter were successfully obtained with the assistance of templating agent, $\mathrm{Mo}_{7} \mathrm{O}_{24}{ }^{6-}$, at room temperature. XRD, TEM, UV-DRS, XPS, and BET characterization were used to measure the samples. The product has a moderately high surface area of $132 \mathrm{~m}^{2} \mathrm{~g}^{-1}$ and a narrow mesoporous structure with an average pore diameter of $3.5 \mathrm{~nm}$. The methyl orange (MO) aqueous solution was used to evaluate the photocatalytic capability of the mesoporous $\mathrm{SnO}_{2}$ under UV light irradiation.

In "Production of biologically activated carbon from orange peel and landfill leachate subsequent treatment technology," orange peel activated carbon with biophile characteristic has been fabricated by one-step method. The yield of the as-prepared biological activated carbon is $50.9 \%$, accompanied with the BET specific surface area, and pore volume reached $1477 \mathrm{~m}^{2} / \mathrm{g}$ and $2.090 \mathrm{~m}^{3} / \mathrm{g}$, respectively. The orange peel active carbon has hydroxyl, carbonyl, methoxy, and lactones hydrophilic functional group. The orange peel active carbon had a well adsorption capability to deal with leachate biochemical tail water, ascribed to small crystallite size and crystallite thickness. The removal rate of $\mathrm{UV}_{254}$ is $63.0 \%$ and TOC is $59.7 \%$, respectively. This research can advance the process of effectively controlling water pollution, improving area water quality, easing orange peel waste pollution, and promoting coordinated development among society, economy, and environment.

The paper "Removing and recovering phosphate from poultry wastewater using amorphous ceramics" described that a novel strategy for phosphate from poultry wastewater was developed using amorphous ceramics. Widely available, inexpensive materials such as lime and silica fume were used to synthesize amorphous ceramics, which showed high performance for phosphate removal and recovery from poultry wastewater. Phosphate content on the surface of amorphous ceramics could reach $14.20 \%$. This study may be interesting and promising which can advance the development of 
the phosphate removing and recovering from poultry wastewater.

The paper entitled "Structural and electrochemical properties of lithium nickel oxide thin films" documented that $\mathrm{LiNiO}_{2}$ thin films were fabricated by RF magnetron sputtering. X-ray diffraction and field-emission scanning electron microscopy were used to investigate the microstructure of the films. The electrochemical properties were investigated with a battery cycler using coin-type half-cells. Surface carbonate existed when the $\mathrm{LiNiO}_{2}$ thin films were annealed below $500^{\circ} \mathrm{C}$. The results reflected that surface carbonate interrupted the Li intercalation and deintercalation during charge/discharge. The capacity did not enhance; nevertheless the annealing process enhanced the crystallization of $\mathrm{LiNiO}_{2}$. The $\mathrm{ZrO}_{2}$-coated $\mathrm{LiNiO}_{2}$ thin film showed an enhanced discharge capacity compared to prine $\mathrm{LiNiO}_{2}$ thin film. Hence, the enhancement in electrochemical characteristics can be attributed to the inhibition of surface carbonate by the $\mathrm{ZrO}_{2}$ coating layer.

The paper entitled "Synthesis of an anion-exchange membrane based on imidazolium-type ionic liquids for a capacitive energy extraction Donnan potential device" described that polymeric anion-exchange membranes (AEMs) were synthesized via the photocopolymerization of a vinyl imidazolium ionic liquid, with 1-butyl-3-(4-vinylbenzyl)imidazolium chloride $([\mathrm{BVBI}][\mathrm{Cl}])$ as the anion-exchange group and styrene, acrylonitrile, and divinylbenzene as cross-linked agents. The mechanical, physical, and electrochemical properties of the fabricated AEMs such as swelling degree (\%), water uptake (\%), ion-exchange capacity ( $\mathrm{mmol} / \mathrm{g})$, thermal stability, tensile strength (Mpa), tensile modulus (Mpa), elongation (\%), and ionic conductivity $(\mathrm{S} / \mathrm{cm})$ were evaluated. The obtained AEMs were demonstrated to have good mechanical, physical, and electrochemical properties for application of a capacitive energy extraction Donnan potential (CDP) device membrane. The CDP device was obtained by using the synthesized AEM, and the specific capacitance of the CDP device with AEM was determined as $2.53 \mathrm{~F} / \mathrm{g}$ and $3.44 \mathrm{~F} / \mathrm{g}$ by galvanostatic charge/discharge and cyclic voltammetry, respectively. The synthesized AEM with imidazolium ionic liquid can be beneficial for the development of the CDP device membrane.

The review article "Synthesis and application of onedimensional $\mathrm{La}(\mathrm{OH})_{3}$ nanostructures: an overview" is aimed at describing and summarizing current status on the application of $1 \mathrm{D} \mathrm{La}(\mathrm{OH})_{3}$ nanostructures including nanowires, nanoneedles, nanobelts, and nanorods. Various strategies utilized to synthesize the $1 \mathrm{D} \mathrm{La}(\mathrm{OH})_{3}$ nanostructures are discussed, such as precipitation, composite-hydroxidemediated, hydrothermal, and solvothermal methods, as well as the introduction of the corresponding growth mechanisms. Then, the unique properties of $1 \mathrm{D} \mathrm{La}(\mathrm{OH})_{3}$ nanostructures are assigned to their unique electronic structures and numerous transition modes involving the $4 \mathrm{f}$ shells of these ions are represented. Furthermore, the wide applications in photocatalyst, capacitors, and photoluminescence based on the unique properties are discussed. Finally, the article ends with a summary and some perspectives on the challenges and new directions in this emerging area.

\section{Acknowledgment}

We wish to express our sincere thanks to all the authors for submitting interesting contributions to this special issue.

Fan Dong

Sirilak Sattayasamitsathit

Yu Xin Zhang

Ying Zhou 

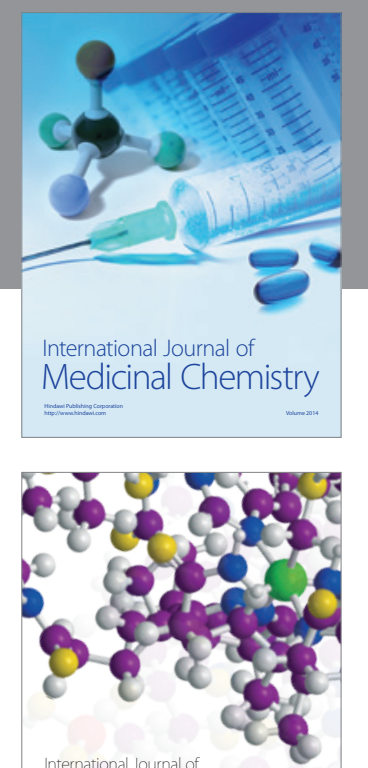

\section{Carbohydrate} Chemistry

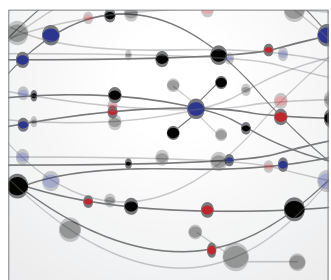

The Scientific World Journal
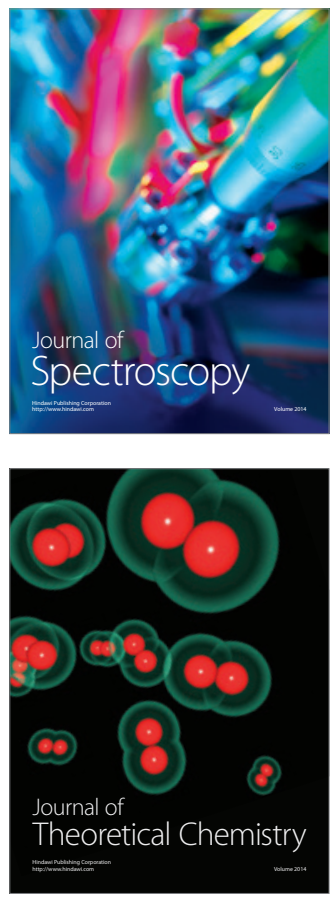
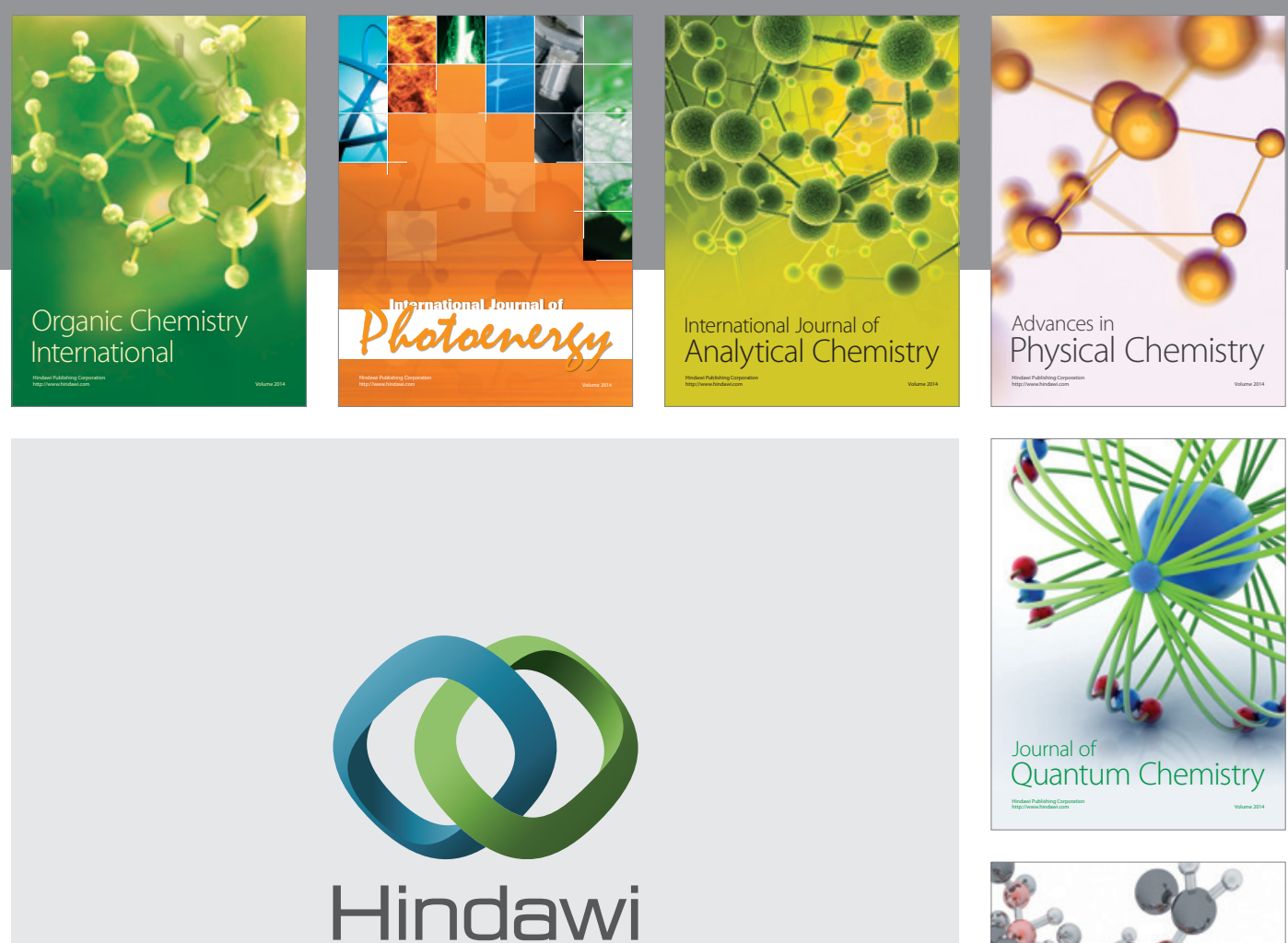

Submit your manuscripts at

http://www.hindawi.com

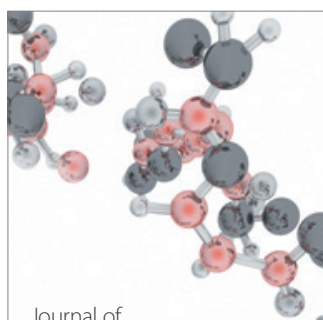

Analytical Methods

in Chemistry

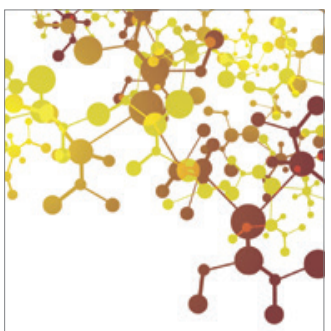

Journal of

Applied Chemistry

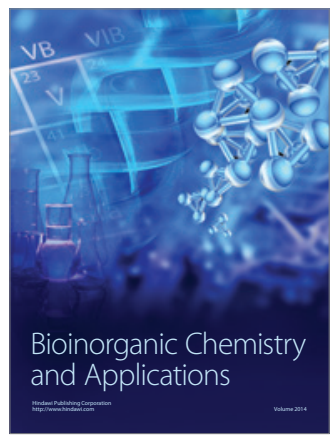

Inorganic Chemistry
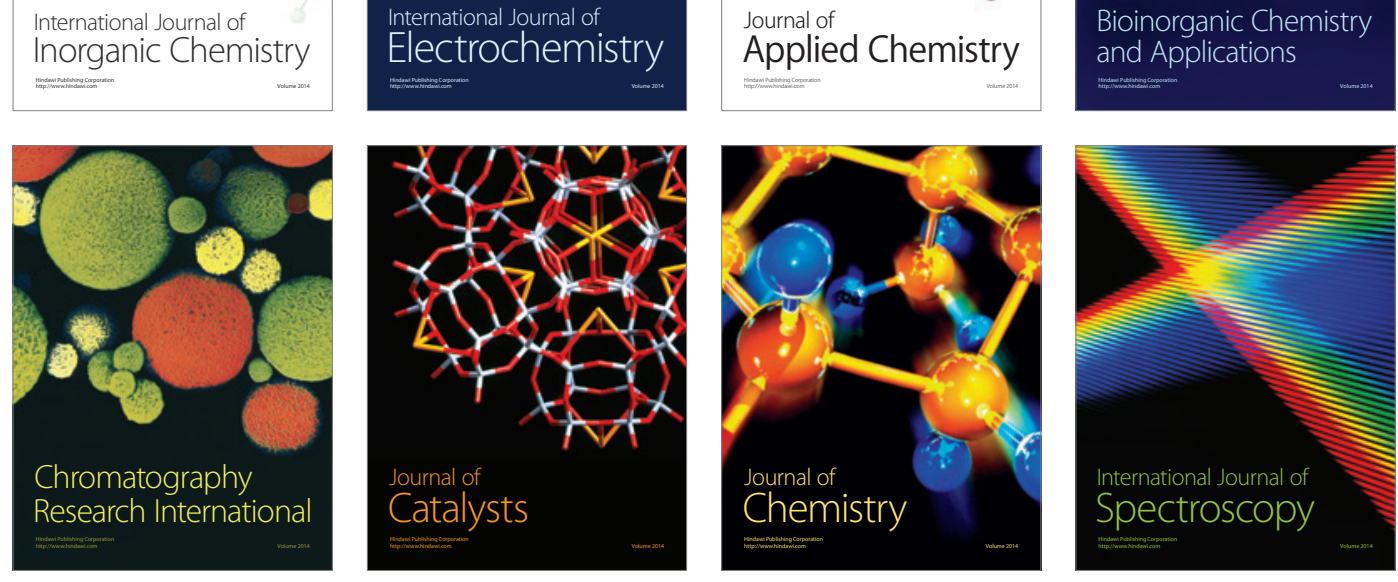